\title{
Neural correlates of focused attention in cognitively normal older adults
}

\author{
Jennifer R. Bowes ${ }^{1}$, Patrick Stroman ${ }^{1,2,3}$, Angeles Garcia ${ }^{1,4}$ \\ ${ }^{1}$ Centre for Neuroscience Studies, Queen's University, Kingston, Canada; \\ ${ }^{2}$ Department of Diagnostic Radiology, Queen's University, Kingston, Canada; \\ ${ }^{3}$ Department of Physics, Queen's University, Kingston, Canada; \\ ${ }^{4}$ Department of Medicine, Queen's University, Kingston, Canada. \\ Email: 6jrb2@queensu.ca
}

Received 28 April 2011; revised 18 May 2011; accepted 22 July 2011.

\begin{abstract}
Focused attention (FA) is among the cognitive functions that decline with aging. The Stroop task was used to investigate the neural correlates underlying FA in cognitively normal older adults. Twenty-one participants underwent a novel functional magnetic resonance imaging ( $F M R I)$ verbal Stroop task paradigm. Colour words were printed in an incongruent ink colour. Series 1 consisted of four blocks "Read the word" followed by four blocks "Say the colour of the ink"; Series 2 alternated between the two conditions. Functional data were analyzed using SPM5 to detect anatomical areas with significant signal intensity differences between the conditions. Within-group analyses of the "Say the colour of the ink" minus "Read the word" contrast yielded significant activation in the left supplementary motor area, bilateral inferior frontal gyrus, bilateral precentral gyrus, left insula and right superior frontal gyrus $(p<0.05$, uncorrected). These results, using verbal responses, are consistent with previous manual modality StroopfMRI studies in older adults. Verbal responses may provide a more suitable modality for older adults and certain patient populations.
\end{abstract}

Keywords: $f$ MRI; Focused Attention; Stroop

\section{INTRODUCTION}

There is a growing interest in studying cognitive domains, such as attention and inhibitory control, which decline during the aging process. Older adults appear to be more susceptible to cognitive interference than $y$ ounger adults [1] and the frontal lobe, which is instrumental in exercising attention control, is susceptible to some of the earliest and more rapid pathological changes that occur in the aging brain [2]. Consequently, pathological changes in the frontal lobe may contribute to the increased susceptibility of older adults to cognitive interference. More specifically, attention plays a critical role in restricting the sensory input load that enters our processing system [3], and attentional control is engaged, most importantly, in times of cognitive conflict [4]. Focused attention (FA) biases the information we are exposed to by enhancing task-relevant information, and is used as a measure of the ability to inhibit irrelevant information [5]. In order to understand the changes that occur with aging, and with diseases such as Alzheimer's Disease (AD), it is therefore very important to understand the changes that occur in the processes that control attention.

The Stroop test [6] is a well-known experimental paradigm that introduces cognitive conflict by presenting colour names printed in a non-matching (incongruent) ink colour, and is considered the gold standard for assessment of FA. Cognitive interference reveals itself in the Stroop task by an increase in response latency and/or higher error rate when naming the ink colour $[1,4]$. However, the information that can be obtained about attentional processes may be significantly enhanced by combining the Stroop task with functional magnetic resonance imaging ( $f$ MRI). Finding the neural substrates that underlie the Stroop effect may advance our understanding of how humans maintain attention and perform the Stroop task [7]. The high spatial resolution and noninvasive nature of $f$ MRI makes it a valuable tool for identifying the brain regions mediating FA. Initial StroopfMRI studies in young adults have provided the foundation to allow further investigations into the functional processes in healthy aging. However, the means by which we study the cognitive processes may require different approaches based on the demographics of those being studied. While Stroop-fMRI studies in young 
adults have employed both verbal and manual response modalities, only the manual button press modality has been used with older adults [8-10]. By both self-report and performance, older adults have more difficulty using the button-box than the young adults [10]. The buttonbox modality adds additional cognitive demands by requiring subjects to remember which button corresponds to which colour stimuli. This modality may add task difficulty disproportionately for older adults.

Just as cognitively normal older adults appear susceptible to cognitive interference, pathological conditions such as $\mathrm{AD}$ interfere with cognitive function that increase susceptibility to cognitive interference. An assessment of cognitive functioning in older adults and certain patient populations, such as those diagnosed with $\mathrm{AD}$ and mild cognitive impairment (MCI), are an important part of clinical care. The Stroop task is among the tools used in a clinical setting to assess cognitive function, specifically FA, but has also been studied extensively in experimental paradigms. If cognitively normal older adults report difficulty during a manual modality Stroop-fMRI paradigm, one might expect that patients with cognitive impairments, such as those diagnosed with $\mathrm{AD}$, would find such a paradigm even more difficult. In order to gain a better understanding of the neural mechanism of FA in these populations, an experimental paradigm suitable to the cognitive abilities of such persons must be applied in order to more accurately ascertain that the cognitive function of FA is being captured. The use of verbal modality in Stroop-fMRI paradigms may be a suitable paradigm that allows one to investigate FA in older adults and in patient populations such as $\mathrm{AD}$ and MCI. In order to extrapolate the findings from experimental studies to the clinical setting, one must minimize the differences in the administration of the Stroop task across the two settings. The use of the verbal response modality in the fMRI task would be consistent with the verbal responses required in the paper version of the Stroop task that is used in clinical settings.

Although many variations of the Stroop task paradigm exist, the majority of Stroop-fMRI studies have instructed subjects to name the ink colour across incongruent, congruent, neutral or non-lexical stimuli to yield brain regions underlying Stroop interference. However, in fMRI, to best determine the brain regions underlying a given cognitive process, the differences between the two conditions being compared should be minimized as to isolate the cognitive process of interest. In the current study, the colour word stimuli presented are identical in all incongruent trials, with the only difference being the instructions given to the subject ("Read the word" or "Say the colour of the ink"). In this way, the current ex- perimental paradigm isolates the cognitive process of FA better than the previous Stroop-fMRI studies in older adults, and in doing so, improves the similarity between the fMRI Stroop task and the original paper version of the Stroop task.

The current study offers a novel Stroop-fMRI paradigm that employed a verbal response modality using incongruent colour-word trials to yield the Stroop effect by contrasting reading the word from saying the ink colour in cognitively normal older adults.

\section{METHODS}

\subsection{Subject Population}

The study was approved by the University Research Ethics Board. All subjects gave written informed consent prior to undergoing any study procedures. Twenty-one cognitively normal, independent community dwelling older adults (13 women, 8 men) were recruited. Subjects were all native or highly proficient English-speaking volunteers between the ages of 60 and 85 years (mean age $\pm \mathrm{SD}, 72 \pm 8$ ), who could safely undergo a MRI, with no colour blindness, neurological or other medical conditions that could interfere with the task protocol.

\subsection{Cognitive Assessment}

Subjects underwent a neuropsychological test battery to assess their general cognitive function including the following tests: the Mini-Mental State Examination (MMSE) [11], the Montreal Cognitive Assessment (MoCA) [12], the Mattis Dementia Rating Scale (DRS) [13], the Stroop test, the Trail Making Test- Part B (TMT-B) [14], and the California Verbal Learning Test-Version II (CVLT-II) [15]. Subjects were considered to have normal cognitive abilities with the following test scores: MMSE $\geq 26$, MoCA $\geq 26$, DRS $\geq 123$, Stroop colourword score of $>65$ and CVLT-II verbal recall sub-scores $>1.5 \mathrm{SD}$ below the mean for age, sex and years of education.

\section{3. fMRI Experimental Task}

The fMRI experiment was carried out at the Queen's University Research MRI Facility. The Stroop test used was an adaptation from the word-colour incongruent task taken from the paper version of the Stroop test. The four colour word stimuli used in the experiment were: blue, green, tan and red. Colour word stimuli were presented visually via back projection onto a screen that was viewed through a mirror attached to the head coil of the scanner. The presentation of the colour word stimuli was run using MatlabR2006b (The Mathworks, Natick, MA). An MR-compatible microphone with noise cancellation was placed in front of the participant's mouth to record the verbal responses to the presented colour 
word stimuli. Subjects were instructed to speak aloud, but quietly, and without excessive enunciation, in an attempt to minimize head motion while speaking. Verbal reminders to keep their head still and limit any body movements were made at short rest intervals between the series, if movement for a given subject was a concern. Subjects were given headphones to wear to reduce the noise of the MR scanner and to provide a means to communicate with the subject throughout the experiment. Padding was placed between the outside of headphones and the sides of the head coil to reduce the opportunity for the subjects to move their head. The experimental protocol was carried out as a block design with four functional series' as displayed in Figure 1. Each block was 28 seconds and consisted of 16 colour word stimuli, each of which was presented for 1.75 seconds. Each block was separated by a 14 -second rest period. Series 0 consisted of 4 blocks with colour word stimuli presented in black ink and participants were instructed to read the word. The instructions "Read the word" preceded the commencement of the first block with a fixation cross displayed during the remaining rest periods. A total of 91 images were acquired with a sequence scan time of approximately 3 minutes. Series 1 and 2 consisted of 8 blocks with colour word stimuli presented in an incongruent ink colour with the rest period preceding each block displaying the instructions for the subsequent block. A total of 175 images were acquired with a sequence scan time of approximately 6 minutes for each Series 1 and 2. In Series 1, participants were instructed to "Read the word" (herein referred to as "Word" condition) in the first 4 blocks, and instructed to "Say the colour of the ink" (herein referred to as "Colour" condition) in the last 4 blocks. In Series 2, blocks alternated between "Word" and "Colour" conditions. Series 2 was performed twice and herein referred to as Series 2a and 2b.

\subsection{Image Acquisition}

All imaging was acquired on a 3 Tesla Siemens Magnetom Trio MRI system (Siemens Medical Systems, Erlangen, Germany) in conjunction with a 12-channel head coil. Motion correction software was used during scanning, and in the subsequent data analysis. Structural and functional sequences were acquired. A 176 slice, highresolution anatomical scan was acquired with a T1weighted 3D, MP-RAGE sequence (single shot, ascending sequence in the sagittal plane with TR $=1760 \mathrm{msec}$, $\mathrm{TE}=2.2 \mathrm{msec}$, flip angle $=9^{\circ}, \mathrm{FoV}=256 \mathrm{~mm}$, voxel size $=1 \mathrm{~mm}^{3}$ ). Functional data was acquired along the anterior commissure-posterior commissure (AC-PC) line with blood oxygenation level dependent (BOLD) $\mathrm{T}_{2}{ }^{*}$ weighted echo planar imaging. Thirty-two axial slices (TR $=2000 \mathrm{msec}, \mathrm{TE}=30 \mathrm{msec}$, flip angle $=78^{\circ}, \mathrm{FoV}=$ $211 \mathrm{~mm}$, voxel size $=3.3 \mathrm{~mm}$ cubic voxels) were acquired providing whole-brain coverage using interleaved acquisition.

\subsection{Data Analysis}

Statistical analysis on demographics, cognitive assessment and performance accuracy was performed using SPSS, Version 17.0 (SPSS Inc., Chicago, IL). Subject's verbal responses to colour word stimuli were recorded for analysis of performance accuracy on the StroopfMRI task. Condition blocks with less than $75 \%$ accuracy were excluded from $f M R I$ analysis. Errors were classified as incorrect, missed or corrected responses.

Spatial pre-processing and statistical analysis of the imaging data was carried out using SPM5 (Wellcome Department of Cognitive Neurology, University College

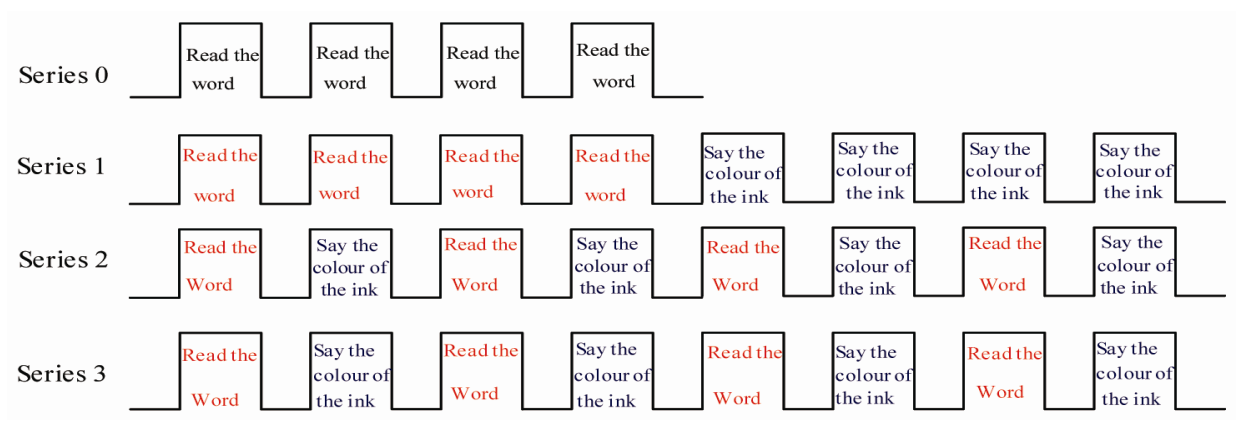

Figure 1. Stroop-fMRI experimental paradigm. In Series 0, 4 blocks of colour word stimuli were presented in black ink and participants were asked to read the word. The first rest period displayed the instruction of "Read the word"; the remaining rest periods displayed a fixation cross. In Series 1, 2a, and $2 \mathrm{~b}$ presented 8 blocks of colour word stimuli presented in an incongruent ink colour, with the rest period displaying the instructions to the subsequent condition block. In Series 1, participants were asked to 'Read the word' for the first 4 blocks, and to "Say the colour of the ink" for the last 4 blocks. Series $2 \mathrm{a}$ and $2 \mathrm{~b}$ are identical, with blocks alternated between the "Read the word" and "Say the colour of the ink" conditions. 
London, England). The first two functional scans in each series were discarded from the analyses to allow the longitudinal magnetization to reach a steady state. The following pre-processing steps were carried out: realignment, co-registration, segmentation, normalization and smoothing. First, functional $\mathrm{T}_{2}{ }^{*}$-weighted images were spatially realigned to the first analyzed volume scanned in the run using a six-parameter rigid-body transformation to compute a mean functional image. Next, the high resolution $\mathrm{T}_{1}$-weighted structural scan was co-registered to the mean realigned functional images. SPM5 combines segmentation, spatial normalization and bias correction in a unified model approach [16]. The segmented images were then normalized into the standard stereotactic space of the Montreal Neurological Institute (MNI) template. Lastly, the functional images were smoothed with a Gaussian kernel of $8 \mathrm{~mm} \times 8 \mathrm{~mm} \times 8 \mathrm{~m}$ full width at half maximum. A 128-second temporal high pass filter (with $1 / 128$ seconds $(0.0078 \mathrm{~Hz})$ as the frequency cut off) was applied to the functional data to exclude low frequency fluctuations and artifacts. Statistical analysis was performed using the univariate approach of the general linear model [17] whereby the box-car reference function is convolved with the hemodynamic response function to account for the delay in the BOLD signal. For each subject, a design matrix was created with the rest periods, correct "Word" blocks and correct "Colour" blocks applied as regressors, in addition to the six realignment parameters. Error blocks were represented as separate regressors in the design matrix, though not used in any contrasts. Voxel-wise statistical analysis was then carried out to determine which voxels were activated by the different experimental conditions. Fixedeffects analysis was applied to each participant resulting in statistical parametric maps (SPMs) for every contrast of interest, using a significance threshold of $p=0.001$, uncorrected for multiple comparisons. Individual SPMs from each participant were then pooled together and entered into a random-effects analysis. A one- sample $t$-test was applied to investigate the within-group activation level with a significance threshold of $p=0.05$, uncorrected for multiple comparisons. To control for false positives, a spatial extent cluster threshold of 3 voxels was applied. The coordinates of voxels that survived the statistical threshold were entered into MRICro software [18], which uses an automated anatomical labeling (AAL) template [19] to report the localization of the activation. Significant voxels reported in SPM5 are reported in MNI space, and the AAL template was created based on the anatomical parcellation of the spatially normalized single-subject, segmented high resolution T1 volume provided by MNI. Realignment parameters of motion-corrected and non motion-corrected functional data were used to estimate subject motion during the experimental task.

\section{RESULTS}

\subsection{Behavioural Results}

Demographic descriptives and mean neuropsychological test battery scores are listed in Table $\mathbf{1}$. In the paper version of the Stroop task administered as part of the test battery, all subjects were significantly better reading the word than naming the colour of the ink $(p<0.001)$.

Both non-motion corrected and motion corrected data were viewed to inspect subject motion parameters. The average subject movement was estimated based on the rotation about and the translation along each of the 3 coordinate axes $(x, y$, and $z)$. There was a mean $( \pm \mathrm{SD})$ translation of $2.85 \pm 1.193 \mathrm{~mm}$ and a rotation of $2.964 \pm$ 1.454 degrees for non-motion-corrected acquired images. For the motion corrected acquired images, the mean ( \pm SD) translation was $1.786 \pm 0.681 \mathrm{~mm}$ and a rotation of $1.905 \pm 0.785$ degrees.

Performance accuracy during the Stroop-fMRI experiment was assessed. The mean $( \pm S D)$ numberof total errors across all series was $8.00 \pm 6.834$. The mean $( \pm$ $\mathrm{SD})$ number of errors in Series 1 , Series 2a and Series $2 \mathrm{~b}$ were $1.38 \pm 2.397,3.57 \pm 4.976$ and $3.05 \pm 4.511$, respectively. There were no significant differences in the numbers of errors between Series 1 and 2a ( $p=0.107)$, Series 1 and $2 \mathrm{~b}(p=0.144)$ and Series $2 \mathrm{a}$ and $2 \mathrm{~b}(=$ $0.725)$. The mean $( \pm S D)$ numbers of errors in each of the "Word" and "Colour" conditions were $2.90 \pm 3.961$ and $4.86 \pm 4.983$, respectively. Across all of the series, there

Table 1. Demographic descriptives and mean neuropsychological test battery scores.

\begin{tabular}{cc}
\hline & $\begin{array}{c}\text { Cognitively Normal Older } \\
\text { Adults } N=21 \text { Mean (SD) }\end{array}$ \\
\hline Gender (M:F) & $8: 13$ \\
Age (years) & $71.24(5.682)$ \\
Education (years) & $16.10(4.170)$ \\
MMSE & $29.62(0.498)$ \\
MoCA & $27.00(1.949)$ \\
DRS & \\
Total Score & $141.90(2.047)$ \\
Stroop & $112.00(0.000)$ \\
Word Score & $96.24(11.528)$ \\
Colour Score & $86.00(20.039)$ \\
TMT-B (seconds) & \\
CVLT-II & $10.191(3.750)$ \\
SDFR Raw Score & $10.429(3.749)$ \\
LDFR Raw Score
\end{tabular}

M = Male; F = Female; MMSE = Mini-Mental State Examination; MoCA = Montreal Cognitive Assessment; DRS = Dementia Rating Scale; TMT-B= Trail Making Test-Part B; CVLT-II = California Verbal Learning TestVersion II; SDFR = Short Delay Free Recall; LDFR = Long Delay Free Recall; ${ }^{*} N=20$. 
were no significant differences in error rates between the "Word" and "Colour" conditions ( $p=0.144)$. When the number of errors by condition is stratified by series, as seen in Figure 2, only Series 1 showed significantly more errors in the "Colour" condition than in the "Word' condition ( $p=0.026)$. The mean $( \pm \mathrm{SD})$ number of incorrect, corrected, and missed responses were $1.286 \pm$ 2.825, $0.794 \pm 1.894,0.587 \pm 1.116$, respectively.

\subsection{Imaging Results}

The main contrast of interest was the incongruent "Colour" condition against the "Word" condition yielding brain areas more activated in the "Colour" than in the "Word" condition. The within-group analysis of the interference condition across Series 1, 2a and 2b yielded greater activation in the left supplementary motor area (SMA), bilateral precentral gyrus, right pars opercularis of the inferior frontal gyrus (IFG), left insula, and right supe rior frontal gyrus ( $p=0.05$, uncorrected for multiple comparisons) as listed in Table 2. The brain areas significantly more active in the "Colour" condition compared to the "Word" condition are displayed on the SPM

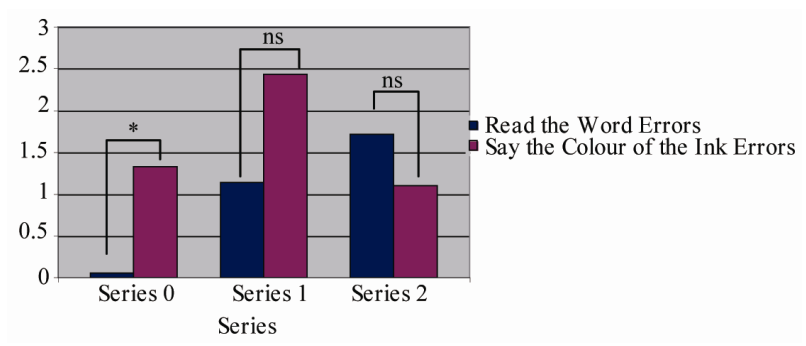

Figure 2. Mean number of errors by condition type across the incongruent series. Mean number of errors by condition type across the incongruent series (Series 1 , Series $2 \mathrm{a}$ and Series $2 \mathrm{~b}$ ) for the cognitively normal older adults. There was a significant difference in the number of errors between the "Read the word" and "Say the colour of the ink" condition for Series 1 ( $p$ $=0.026)$, but no significant differences between conditions in either Series $2 \mathrm{a}(p=0.221)$ or Series $2 \mathrm{~b}(p=0.436) .\left({ }^{*}=\right.$ statistically significant; $n s=$ not significant $)$.

Table 2. Brain areas significantly more active in the "Colour" condition than in the "Word" condition from the within-group analysis of the cognitively normal older adult group.

\begin{tabular}{|c|c|c|c|c|c|c|}
\hline \multirow{3}{*}{ Region } & \multicolumn{6}{|c|}{ MNI coordinates in talarach } \\
\hline & \multirow{2}{*}{ Side } & \multicolumn{3}{|c|}{ Space $(\mathrm{mm})$} & \multirow{2}{*}{$T$} & \multirow{2}{*}{ Z-score } \\
\hline & & $x$ & $y$ & $Z$ & & \\
\hline \multirow{2}{*}{$\begin{array}{l}\text { Supplemerntary } \\
\text { motor area }\end{array}$} & $\mathrm{L}$ & -9 & 9 & 48 & 3.89 & 3.32 \\
\hline & I & -42 & 3 & 27 & 3.20 & 2.84 \\
\hline \multirow[t]{2}{*}{ Precertral gyrus } & L & -36 & 0 & 36 & 3.15 & 2.81 \\
\hline & $\mathrm{R}$ & 60 & 9 & 39 & 2.78 & 2.53 \\
\hline \multirow{2}{*}{$\begin{array}{l}\text { Inferior frortal } \\
\text { gyrus, pars oper- } \\
\text { cularis }\end{array}$} & 48 & 48 & 18 & 6 & 2.62 & 2.40 \\
\hline & $\mathrm{L}$ & -27 & 21 & 6 & 2.50 & 2.40 \\
\hline Insula Superior & $R$ & 24 & 12 & 69 & 2.43 & 2.25 \\
\hline frontal gyrus & $\mathrm{K}$ & 27 & 3 & 66 & 1.97 & 1.87 \\
\hline
\end{tabular}

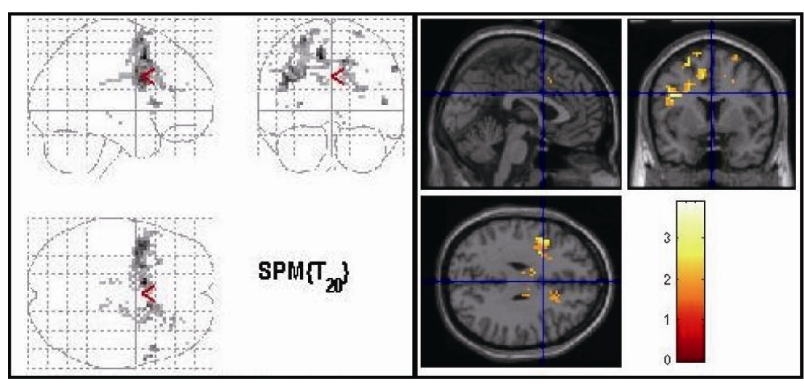

Figure 3. Overlay of brain activation of the within-group analysis for the "Say the colour of the ink" minus "Read the word" contrast. An overlay of brain regions on the SPM glass brain (left) and on an anatomical template (right) showing increased activity in the "Say the colour of the ink" than in the incongruent "Read the word" by the cognitively normal older adult group ( $p<0.05$, uncorrected).

in Figure 3 ( $p=0.05$, uncorrected for multiple comparisons). The main contrast "Colour" minus "Word" for Series 1 yielded significant activation in the left pars opercularis of the IFG, right insula, left inferior and superior parietal cortex, left lingual, right middle and superior frontal gyrus and the right precentral gyrus. For the Series 1 main contrast of interest, only the left pars opercularis of the IFG survived the statistical threshold of $p$ $=0.001$. There were no brain regions for either Series 2a or $2 \mathrm{~b}$ main contrast of interest that survived a $p=0.001$ statistical threshold. The contrast comparing Series 0 "Word" condition with Series 0 rest condition (fixation cross) yielded significant brain activation in expected speech production and reading brain regions, including the precentral gyrus, SMA, middle frontal gyrus, and lingual gyrus with predominantly left hemisphere activation.

\section{DISCUSSION}

This is the first study to investigate the neural correlates of FA using a Stroop- $f$ MRI task paradigm in cognitively normal older adults using verbal responses rather than a manual button press modality. The main contrast of interest for the incongruent trials, "Colour" minus the "Word", elicited increased activation including the SMA, precentral gyrus, and IFG. The results of the current study are consistent with brain regions activated in previous manual button press modality Stroop- $f$ MRI studies in older adults [8-10]. Similar brain regions within the neural network of FA of the current verbal modality study with previously published manual modality studies, suggest the current study's novel verbal modality StroopfMRI task offers a suitable paradigm to investigate the neural correlates of FA. Furthermore, the task was performed well by the cognitively normal older adults.

\subsection{Behavioural Results}

In the paper version of the Stroop test administered as 
part of the neuropsychological test battery, as expected, subjects were significantly better at reading the word than naming the colour of the ink. Interestingly, in the Stroop-fMRI experimental task, when errors were collapsed across all series, subjects did not make significantly more errors in the "Colour" condition than in the "Word" condition. This is likely a reflection of the experimental design, although overall, very few errors were made during the Stroop-fMRI task. In Series 1, which consisted of four blocks of "Word" followed by four blocks of "Colour", subjects made significantly more errors when naming the ink colour. Whereas, in Series 2a and 2b, which consisted of alternating "Word" and "Colour" conditions, there was no significant difference in errors between the two conditions, and may reflect the difficulty subjects had in remembering which set of instructions was given. The majority of the "Word" errors were made in the alternating Series 2a and 2b. That is, prior to the introduction of the "Colour" condition, reading the word is a fairly automatic process for the subjects. However, once the "Colour" condition is introduced, reading the word becomes less automatic, and FA is likely required for both conditions because what is considered the irrelevant colour dimension, changes each block.

\subsection{Imaging Results}

The main contrast of interest for the current study was the subtraction of the incongruent "Word" condition from the incongruent "Colour" condition. Significant activation was observed in the left SMA, bilateral precentral gyrus, and right pars opercularis of the IFG, left insula and right superior frontal gyrus. The neuroimaging results of the study are in keeping with the results from previous Stroop-fMRI studies of older adults. Common activated regions between the work of Zysset et al. [9] and the current findings include the IFG and superior frontal gyrus. In the Stroop-fMRI study of Milham et al. [8], older adults activated a network of structures including the IFG, as found in the current study, and the SMA showed increased activity specific to the presence of conflicting colour information. The study's finding of right frontal gyri was also found in the Stroop-fMRI study of Langenecker et al. [10].

Similar regions of brain activation were found between the current study and previous Stroop-fMRI studies of older adults despite methodological differences. Chief among the methodological differences was the use of a verbal response modality in this study. The greatest concern with use of verbal responses in an experimental paradigm is the increased susceptibility to motion artifact inherent with verbal responses. However, means to account for and correct some of the movements made by subjects during the experimental task were undertaken to minimize subject motion and to minimize its effect on the imaging data in the present study, as described in the Methods section. Motion-corrected software, which included the automated image registration for motion correction of $\mathrm{fMRI}$ time-series during the pre-processing of the functional data, was also applied to reduce some of the effects caused by changes in position between successive images. Although uncorrected motion can confound functional data by causing signal changes, it is those voxels that lie close to tissue boundaries that are most susceptible [20]. Given our knowledge of the neural substrates that underlie the Stroop task, the brain regions expected to be involved with this task are, for the most part, not in close proximity to areas of high contrast or tissue boundaries. Analyzing the motion parameters and visually inspecting the motion data allows one to consider the influence the motion has on the functional results. Subject inter-scan movement can be estimated using the motion parameters obtained from the spatial realignment step in pre-processing, whereby each image in the time series is compared to the first, reference image of that time series. The time series images of translation and rotation motion parameters were visually inspected to exclude any subject with excessive motion. Given the verbal modality of the experimental task, excessive motion was considered if both translation and rotation measures were $>5 \mathrm{~mm}$ and degrees, respectively. Using this cut-off criterion, no subjects were excluded from the analysis. The motion-corrected functional data were used in subsequent analyses and likely reflects the influence of subject motion on the imaging results. However, the non-motion corrected data provides the best estimate of subject motion during the experimental run. By decreasing the length of the experimental paradigm and by giving more reminders to subjects to remain still, the motion parameters for the older adults may be lowered to improve the integrity of the imaging results.

The current study had a larger older adult sample size than previous Stroop-fMRI studies by Milham et al. [8] and Langenecker et al. [10]. The mean age of the older adults in the current study (71 years) was comparable to the mean age of older adults in the Langenecker et al. [10] study (71.1 years) and just slightly older than those in the Milham et al. [8] study (68 years). Among the network of brain regions commonly activated by older adults are the anterior cingulate cortex (ACC)/pre-supplementary motor area (pre-SMA), middle, superior and inferior frontal gyri, and superior and inferior parietal lobules. Older adults have shown increased activation, predominantly in the left hemisphere, in the prefrontal cortex, pre-SMA and left inferior frontal gyrus relative 
to younger adults. These results suggest that older adults rely on, and recruit additional brain areas, particularly prefrontal regions during Stroop task performance [21]. Furthermore, Milham et al. [8], suggested that the parietal cortex has greater involvement in younger than older adults, with older adults reliant on the more anterior, frontal brain regions. This is supported by the findings of the current study where the majority of brain regions underlying Stroop performance lay within the frontal cortex. Implementation of cognitive control may rely on modulation of bilateral inferior frontal regions [22] with activation of the left inferior frontal region consistently found in Stroop-fMRI studies. The inferior frontal region may reflect phonology as this area becomes more active when viewing words and during the retrieval, selection, maintenance or evaluation of semantic knowledge [23]. The inferior frontal region is also home to Broca's area for speech production, and activation of this region in Stroop interference may reflect the response selection competition involving speech articulatory processes [24]. Stroop interference appears to be mediated by competing subvocal articulatory responses in both manual and verbal response modalities suggesting this mediation is not specific to overt speech paradigms [24]. In a positron emission tomography (PET) study, only the left IFG was consistently activated during the interference condition [25]. The left inferior precentral sulcus is located adjacent to the pars opercularis of the IFG and is thought to play a critical role in processing incongruent Stroop stimuli $[24,25]$. The precentral gyrus may correspond to the frontal eye fields [26], which have been shown to be an important area in top-down allocation of attention [27]. The SMA, important for the initiation and control of motor and speech functions [28], and the pre-SMA, involved in encoding and selection of word information [29], are regions found within the medial frontal cortex and have been found to be activated in numerous StroopfMRI studies [8,10,30,31]. Located within the pre-SMA region is the superior frontal gyrus, which is also frequently activated in the incongruent Stroop condition [8, 10,30,32]. According to Taylor et al. [25], the cingulate sulcus/SMA region is involved in resolving response conflict. Brain regions involved in response generation include the cerebellum, SMA and precentral gyri [32]. Given the current Stroop task whereby subjects are required to initiate a verbal response, the activation of the SMA and precentral gyri support the role these regions may play in response generation. The insula is believed to be involved in verbal working memory and selective attention tasks [7] and contributes to cognitive control [9].

Among the early neuroimaging studies of the Stroop task, the Stroop effect almost unanimously activated the
ACC. However, as the Stroop task became used in more studies, and with variations in its paradigm used, the ACC was not consistently activated. This lead Mead et al. [24] to suggest that demonstrating ACC activation may depend on methodological factors in the experimental design. In the current study, the main contrast of interest for Stroop interference did not detect activity within the ACC. Contrasts comparing incongruent colour words to non-lexical stimuli such as colour blocks, hatches or crosses have shown more consistent ACC activation while contrasts with lexical stimuli, as in the current study, have resulted in less frequent ACC activation [24]. Some authors have postulated that the attentional demands are similarly enhanced on incongruent and congruent trials [33], or that the ACC was activated at approximately the same intensity across the incongruent, congruent, and neutral conditions [24]. Furthermore, Milham et al. [8] showed that for older adults, the mere presence of competing colour information of incongruent trials was enough to produce increases in the ACC. The current experimental paradigm consisted largely of incongruent trials, so if the presence of incongruent trials alone produces ACC activation, contrasting the two incongruent conditions will not reveal the common ACC activity in the two conditions. In addition, lesion studies have demonstrated that in some patients with extensive ACC damage, their Stroop performance was unimpaired [21]. These results suggest that although the ACC may be involved in mediating the Stroop effect, it appears that Stroop task performance does not require its activation to resolve the cognitive conflict presented by the Stroop paradigm.

The current study used verbal responses and contrasted incongruent trials of "Read the word" from "Say the colour of the ink" conditions in a Stroop-fMRI task paradigm that more closely mimics the paper, clinical administration of the Stroop task, and whose use of a verbal modality, may be better suited to an older adult population. Changes in cognitive function occur with normal aging, but are exacerbated in pathological diseases such as AD. Attentional deficits, including impairments in FA, have been observed in patients with $\mathrm{AD}$ and MCI [34]. Functional imaging studies with AD and MCI patients would allow for the study of the neural mechanisms underlying their reduced performance on FA tasks such as the Stroop task. However, therein lies the challenge of creating an experimental paradigm to investigate the neural correlates of FA, that patients with diminished cognitive functioning can comprehend and perform as instructed. The Stroop-fMRI task was performed well by the older adults, and suggests that this experimental paradigm, with the use of verbal responses instead of the more complicated manual responses, may 
allow the neural correlates of FA in patients with pathological diseases, such as $A D$ to be investigated.

\section{CONCLUSIONS}

Combined with preventative measures to minimize motion and its effects on the resulting image data, verbal responses may offer a more appropriate modality for use in an older adult population. The novel Stroop experimental paradigm employed in the current study found similar regions of activation than previous Stroop-fMRI studies of older adults using the manual response modality, suggesting that despite a difference in modality used, the results reveal the neural correlates of FA. Furthermore, the verbal task paradigm used in this study may also be useful in studying the neural correlates of FA in patient populations with known impairments of FA. The use of incongruent colour words stimuli across both experimental conditions of the study may provide a means to identify brain areas supporting FA, beyond those, such as the ACC, which may activate upon the presentation of conflicting information of the incongruent word stimuli.

\section{ACKNOWLEDGMENT}

The study was supported by a Queen's University CTAQ Endowment Fund grant.

\section{REFERENCES}

[1] Spieler, D.H., Balota, D.A. and Faust, M.E. (1996) Stroop performance in healthy younger and older adults and in individuals with dementia of the Alzheimer's type. Journal of Experimental Psychology: Human Perception and Performance, 22, 461-479.

doi:10.1037/0096-1523.22.2.461

[2] Whelihan, W.M. and Lesher, E.L. (1985) Neuropscyhological changes in frontal functions with aging. Developmental Neuropsychology, 1, 371-380. doi:10.1080/87565648509540321

[3] Johnson, A. and Proctor, R.W. (2004) Attention theory and practice. SAGE Publications, Inc., Thousand Oak

[4] Kane, M.J. and Engle, R.W. (2003) Working-memory capacity and the control of attention: The contribution of goal neglect, response competition, and task set to Stroop interferences. Journal of Experimental Psychology General, 132, 47-70. doi:10.1037/0096-3445.132.1.47

[5] Townsend, J., Adamo, M. and Haist, F. (2006) Changing channels: An fMRI study of aging and cross-modal attention shifts. NeuroImage, 31, 1682-1692. doi:10.1016/j.neuroimage.2006.01.045

[6] Stroop, O.R. (1935) Studies of interference in serial verbal reaction. Journal of Experimental Psychology, 18, 643-662. doi:10.1037/h0054651

[7] Leung, H.-C., Skudlarski, P., Gatenby, J.C., Peterson, B. S. and Gore, J.C. (2000) An Event-related functional MRI study of the stroop color word interference task. Cerebral Cortex, 10, 552-560.

doi:10.1093/cercor/10.6.552
[8] Milham, M.P., Erikson, K.I. Banich, M.T., Kramer, A.F., Webb, A., Wszalek, T. and Cohen, N.J. (2002) Attentional control in the aging brain: Insights from an $\mathrm{fMRI}$ Study of the stroop task. Brain and Cognition, 49, 277296. doi:10.1006/brcg.2001.1501

[9] Zysset, S., Schroeter, M.L., Neumann, J. and Von Cramon, D.Y. (2007) Stroop interference, hemodynamic response and aging: An event-related fMRI study. Neurobiology of Aging, 28, 937-946. doi:10.1016/j.neurobiolaging.2006.05.008

[10] Langenecker, S.A., Nielson, K.A. and Rao, S.M. (2004) $f$ MRI of healthy older adults during Stroop interference. NeuroImage, 21, 192-200. doi:10.1016/j.neuroimage.2003.08.027

[11] Folstein, M., Folstein, S. and McHugh, P.R. (1975) Mini-mental state: A practical method for grading the cognitive state of patients for the clinician. Journal of Psychiatry Research, 12, 189-198. doi:10.1016/0022-3956(75)90026-6

[12] Nasreddine, Z.S., Phillips, N.A., Bédirian, V., Charbonneau, S., Whitehead, V., Collin, I., Cummings, J.L. and Chertkow, H. (2005) The montreal cognitive assessment, MoCA: A brief screening tool for mild cognitive impairment. Journal of the American Geriatrics Society, 53, 695-699. doi:10.1111/j.1532-5415.2005.53221.x

[13] Mattis, S. (1988) Dementia rating scale. Professional manual. Psychological Assessment Resources, Odessa.

[14] Army Individual Test Battery (1944) Manual of directions and scoring. War Department, Adjunct General's Office, Washington, DC

[15] Delis, D.C., Kramer, J.H., Kaplan, E. and Ober, B.A. (1987) California verbal learning test: Adult version manual. The Psychological Corporation, San Antonia.

[16] Ashburner, J. and Friston, K.J. (2005) Unified segmetation. Neuroimage, 26, 839-851. doi:10.1016/j.neuroimage.2005.02.018

[17] Friston, K.J., Holmes, A.P., Worsley, K.J., Poline, J.P., Frith, C.D. and Frackowiak, R.S.J. (1994) Statistical parametric maps in functional imaging: A general linear approach. Human Brain Mapping, 2, 189-210. doi:10.1002/hbm.460020402

[18] Rorden, C. and Brett, M. (2000) Stereotaxic display of brain lesions. Behavioural Neurology, 12, 191-200.

[19] Tzourio-Mazoyer, N., Landeau, B., Papathanassiou, D., Crivello, F., Etard, O., Delcroix, N., Mazoyer, B. and Joliot, M. (2002) Automated anatomical labeling of activations in SPM using a macroscopic anatomical parcellation of the MNI MRI single-subject brain. NeuroImage, 15, 273-289. doi:10.1006/nimg.2001.0978

[20] Johnstone, T., Ores Walsh, K.S., Greischar, L.L., Alexander, A.L., Fox, A.S., Davidson, R.J. and Oakes, T.R. (2006) Motion correction and the use of motion covariates in multiple-subject $f$ MRI analysis. Human Brain Mapping, 27, 779-788. doi:10.1002/hbm.20219

[21] Adleman, N.E., Menon, V., Blasey, C.M., White, C.D., Warsofsky, I.S., Glover, G.H. and Reiss, A.L. (2002) A developmental fMRI study of the Stroop color-word task. NeuroImage, 16, 61-75. doi:10.1006/nimg.2001.1046

[22] Egner, T. and Hirsh, J. (2005) The neural correlates and functional integration of cognitive control in a Stroop task. Neuroimage, 24, 539-547. doi:10.1016/j.neuroimage.2004.09.007 
[23] Banich, M.T., Milham, M.P., Jacobson, B.L., Webb, A., Wszalek, T., Cohen, N.J. and Kramer, A.F. (2001) Attentional selection and the processing of task-irrelevant information: Insights from $f M R I$ examinations of the Stroop task. Progress in Brain Research, 134, 459-470. doi:10.1016/S0079-6123(01)34030-X

[24] Mead, L.A., Mayer, A.R., Bobholz, J.A., Woodley, S.J., Cunningham, J.M., Hammeke, T.A. and Rao, S.M. (2002) Neural basis of the Stroop interference task: Response competition or selective attention? Journal of the International Neuropsychological Society, 8, 735-742. doi:10.1017/S1355617702860015

[25] Taylor, S.F., Korblum, S., Lauber, E.J., Minoshima, S. and Koeppe, R.A. (1997) Isolation of specific interference processing in the Stroop task: PET activation studies. NeuroImage, 6, 81-92. doi:10.1006/nimg.1997.0285

[26] Paus, T. (1996) Location and function of the human frontal eye-field: A selective review. Neuropsychologia, 34, 475-483. doi:10.1016/0028-3932(95)00134-4

[27] Corbetta, M. (1998) Frontoparietal cortical networks for directing attention and the eye to visual locations: Identical, independent, or overlapping neural systems. Proceedings of the National Academy of Sciences, USA, 95, 831-838. doi:10.1073/pnas.95.3.831

[28] Riecker, A., Mathiak, K., Wildgruber, D., Erb, M., Hertrich, I., Grodd, W. and Ackermann, H. (2005) fMRI reveals two distinct cerebral networks subserving speech motor control. Neurology, 64, 700-706. doi:10.1212/01.WNL.0000152156.90779.89
[29] Alario, F., Chainay, H., Lehericy, S. and Cohen, L. (2006) The role of the supplementary motor area (SMA) in word production. Brain Research, 1076, 129-143. doi:10.1016/j.brainres.2005.11.104

[30] Derrfuss, J., Brass, M., Neumann, J. and von Cramon, D. Y. (2005) Involvement of the inferior frontal junction in cognitive control: Meta-analyses of switching and Stroop studies. Human Brain Mapping, 25, 22-34. doi:10.1002/hbm.20127

[31] Banich, M.T., Milham, M.P., Atchley, R., Cohen, N.J., Webb, A., Wszalek, T., Kramer, A.F., Liang, Z., Wright, A., Shenker, J. and Magin, R. (2000). fMRI studies of Stroop tasks reveal unique roles of anterior and posterior brain systems in attentional selection. Journal of Cognitive Neuroscience, 12, 988-1000. doi:10.1162/08989290051137521

[32] Price, C.J., Moore, C.J. and Frackowiak, R.S. (1996) The effect of varying stimulus rate and duration on brain activity during reading. NeuroImage, 3, 40-52. doi:10.1006/nimg.1996.0005

[33] Melcher, T. and Gruber, O. (2006) Oddball and incongruity effects during Strop task performance: A comparative fMRI study on selective attention. Brain Research, $\mathbf{1 1 2 1}$ 136-149. doi:10.1016/j.brainres.2006.08.120

[34] Parasuraman, R., Greenwood, P.M. and Sunderland, T. (2002) The apolipoprotein E gene, attention and brain function. Neuropsychology, 16, 254-274. doi:10.1037/0894-4105.16.2.254 